\title{
Exotic and not-so-exotic candidates for ultrahigh energy cosmic rays
}

Cite as: AIP Conference Proceedings 566, 295 (2001); https://doi.org/10.1063/1.1378639

Published Online: 04 June 2001

John Swain, L. Anchordoqui, M. T. Dova, T. McCauley, T. Paul, and S. Reucroft

\section{Conference Proceedings}

Get $30 \%$ off all print proceedings!

\section{Enter Promotion Code PDF-30 at checkout}




\title{
Exotic and Not-so-Exotic Candidates for Ultrahigh Energy Cosmic Rays
}

\author{
John Swain ${ }^{*}$,L. Anchordoqui*, M. T. Dova ${ }^{\dagger}$, T. McCauley ${ }^{*}$, \\ T. Paul*, S. Reucroft* \\ ${ }^{*}$ Department of Physics, Northeastern University, Boston, MA 02115 \\ ${ }^{\dagger}$ Universidad Nacional de La Plata, La Plata, Argentina
}

\begin{abstract}
Ultrahigh energy cosmic rays present a major unsolved puzzle. Not only do we not know their origin, but we also have no really solid evidence that they are protons or iron nuclei - the two most popular candidates. We examine briefly a couple of "nonstandard" possibilities: light magnetic monopoles, which are rather exotic, and nuclei heavier than iron, which are perhaps less exotic, but have many attractive features.
\end{abstract}

\section{INTRODUCTION}

The observation of ultrahigh energy cosmic rays (UHECR's) [1] is, at present, a deep mystery. Protons with energies above the GZK [2] cutoff (about $5 \times 10^{19} \mathrm{eV}$ ) lose energy rapidly via inelastic collisions with the cosmic microwave background radiation and thus must come from a nearby source, which seems unlikely. Nuclei, though heavier, are subject to photo-disintegration from red-shifted microwave photons and will lose energy rapidly with distance. Gamma rays of appropriate energy have short mean free paths to create electron-positron pairs and, again, are unlikely candidates. Neutrinos have lower interaction cross-sections, so if they are the particles that make up the highest energy cosmic rays there are a lot of them, and in any case they would likely have to come from the decay of other highly accelerated charged particles. Given the difficulties in identifying a known particle as a candidate, it seems reasonable to turn to exotic candidates. In this paper, we consider the possibility that the highest energy cosmic rays are magnetic monopoles (an exotic possibility) or are very heavy nuclei (a not-so-exotic possibility).

\section{MONOPOLES AS UHECR PRIMARIES}

The idea that UHECR's might be monopoles is an old one due to [3], and revived recently by [4]. There are two considerations that make the monopole hypothesis

CP566, Observing Ultrahigh Energy Cosmic Rays from Space and Earth, edited by H. Salazar, et al. (C) 2001 American Institute of Physics 0-7354-0002-4/01/\$18.00 
attractive: 1) The energy that a monopole with Dirac charge $q_{m}=e / 2 \alpha$ would acquire crossing the $3 \mu$ Gauss galactic magnetic field is about $10^{20} \mathrm{eV}$ - surely an intriguing coincidence at least, and 2) the observed flux of the highest energy cosmic rays is consistent with the Parker bound which requires that there not be so many magnetic monopoles around as to effectively "short out" the galactic magnetic field.

While no reliable direct observations have yet been made of magnetic monopoles, they are attractive objects from a theoretical point of view as the existence of just one automatically implies the quantization of electric charge. Many theories of physics beyond the standard model contain magnetic monopoles naturally; any theory with a simple grand unification (GUT) group that breaks leaving an unbroken $U(1)$ (i.e. electromagnetism) will contain magnetic monopoles with masses around the scale of symmetry breaking. While it is possible to imagine models with lighter monopoles, and indeed even to simply postulate the existence of pointlike Dirac monopoles, direct searches at accelerators pretty much exclude masses below a few tens of GeV. Strict model-independent limits are difficult to set for many reasons including difficulties in treating pointlike monopoles in quantum field theory, and estimating form-factors for non-pointlike monopoles which arise as solitons.

Clearly, magnetic monopoles have electromagnetic interactions. At high energies, a magnetic monopole can be thought of as carrying an electric field with it of $\gamma \vec{v} \times \vec{B}$, where $\gamma$ is the usual Lorentz contraction factor, $\vec{v}$ is the velocity, and $\vec{B}$ is the magnetic field of the monopole at rest. As $\vec{v} \rightarrow c$ (the highly relativistic limit), the monopole then looks like a charge of $Z=1 / 2 \alpha \sim 137 / 2$. (Here and hence we consider the lowest magnetic charge possible). This leads one to think of a relativistic monopole as a minimum-ionizing charged particle depositing about 6 $\mathrm{GeV} /\left(\mathrm{g} \mathrm{cm}^{-2}\right)$, which looks nothing like a high energy proton or nuclear interaction. More promising as signatures are the strong-interaction processes in which a monopole can participate. The generic process is one of

$$
p+\text { Monopole } \rightarrow \ell+\text { Monopole }+X
$$

where $X$ is a collection of other particles with net baryon and lepton number equal to zero and $\ell$ is a lepton. This is an extension of the celebrated "monopole-catalyzed baryon number violation" giving $p \rightarrow \mathrm{e}^{+} \pi^{0}$, but, as we shall see, can be rather more dramatic when the centre-of-mass energy is high.

The cross section expected is a typical strong interaction cross section of about $10^{-26} \mathrm{~cm}^{2}$. The reason for this is the following: the s-wave wavefunction for a charged fermion in the field of a magnetic monopole is infinite at the origin. Any wavefunction then with any admixture of s-wave will then get "sucked into the monopole" with infinite probability.

This infinity, of course, indicates a pathology in the theoretical description of a monopole-fermion interaction, but we can imagine introducing a cutoff at some scale characteristic of the monopole to regularize the result. In the event of a GUT monopole, the scale is the GUT scale, and represents the fact that a GUT monopole is not pointlike, but rather an extended object with length scale the inverse of the 
GUT scale. For a Dirac monopole one might use the Compton wavelength of the monopole, or some other reasonable guess. In any case, the characteristic scale must be much smaller than a proton, so the net result is that the cross section for a proton to interact with a monopole must be given by the proton - the interaction takes place with a cross section typical of the strong interaction.

In the standard model, baryon and lepton number are not conserved [5]. The origin of this phenomenon is the chiral anomaly and the left-right asymmetric electroweak gauge couplings. The fields required are of the form $\vec{E} \cdot \vec{B}$, leading to the idea that one might be able to get baryon and lepton number violation with nothing more than electric and magnetic fields. The problem in the laboratory is that it is hard to get the required field strength, though very high energy particle collider experiments might be able to achieve this sort of effect [6]

In the presence of a monopole, however, the effect is huge. The monopole has a $\vec{B}$ that falls as $1 / r^{2}$ and points radially out. It will effectively suck in a proton (or, rather, a quark from a proton) and in combination with its electric field also falling as $1 / r^{2}$, one gets $\vec{E} \cdot \vec{B} \sim 1 / r^{4}$ and thus the net baryon number change per unit time obtained by integrating the divergences given above is singular, or, more precisely, huge with a cutoff that depends on the details of the monopole.

In GUT's, quarks and leptons appear in the same multiplets. The reason that baryon and lepton number violation don't take place at a noticeable rate is that the GUT group is supposed to be broken to a smaller one which does not mix quarks and leptons. The gauge bosons acquire masses of order the GUT-breaking scale $m_{G U T}$, and all processes they mediate are suppressed by powers of $m_{p} / m_{G U T}$. In this case we have not only the above considerations, but also that a monopole is a topologically nontrivial object. In its centre the Higgs field that broke the GUT symmetry and gave the gauge bosons that correspond to broken directions masses vanishes: all the baryon and lepton number violation processes become unsuppressed there.

Despite the slightly different perspectives of the two approaches to monopoles described above, the net result should be rather model-independent. Using strong interaction cross sections, interactions described above, and the LUND fragmentation model [7], and the AIRES [8] code for particle transport, we have simulated the expected effects of magnetic monopoles and find the following general features:

- The monopoles must be highly relativistic (mass much less than $10^{20} \mathrm{eV}$ ) in order to transfer energy efficiently to nuclei in the atmosphere - otherwise simple kinematics does not allow them to lose much energy

- If the monopoles are very light, they can lose most of their energy in the first collision and generate a proton-like shower.

- If the monopoles are intermediate in mass range, they may initiate several showers, each of somewhat less (again, this depends on kinematics) energy than the previous one making the profile seem more like a stack of proton showers, each started at different depths. 
- With the possibility at each interaction to directly produce a highly energetic neutrino, we expect fluctuations to be larger in monopole-induced showers.

Of course one might also hope that monopole primaries would show some directional preferences as they are accelerated by the galactic magnetic field [9].

\section{VERY HEAVY NUCLEI AS NOT-SO-EXOTIC PRIMARIES}

While the question of whether or not light magnetic monopoles or even magnetic monopoles of any mass exist is still an open one, there is no question that heavy nuclei do exist - could they be possible candidates for ultrahigh energy cosmic rays.

First, on the positive side, a highly charged nucleus is certainly attractive from the point of view of acceleration. In the final analysis, all accelerating mechanisms are electromagnetic and need charge as a "handle" by which to grab onto a particle. The downside is that one expects photodisintegration over relatively short distances via the giant dipole resonances, and one also faces the common belief that there are very few heavy nuclei around which are more massive than iron. Is it possible to find some happy medium ground? We believe the answer may well be yes.

In fact, there is already some observational data which supports the hypothesis of a heavy nucleus primary. The measured density profile of the highest energy Yakutsk event shows a large number of muons which could be the signature of a super-heavy nucleus. In addition the Fly's Eye event, which occurs high in the atmosphere, can be fitted well with a heavy nucleus primary [10]. ${ }^{1}$

Although the abundance of nuclei heavier than iron is expected to be down by 3 to 5 orders of magnitude relative to the ligther ones [12], starbursts may be natural sources able to produce relativistic super-heavy nuclei. These astrophysical environments are supposed to contain large populations of $O$ and Red Giant stars [14], and we believe the supernovae rate is as high as $0.2-0.3 \mathrm{yr}^{-1}$ [15]. Of special interest here, the arrival directions of the Fly's Eye and Yakutsk super-GZK events $\left(b=9.6^{\circ}, l=163^{\circ}\right.$ and $\left.b=3^{\circ}, l=162^{\circ}\right)$ seem to point towards the nearby metallyrich galaxy M82 $\left(b=41^{\circ}, l=141^{\circ}\right)[16]$ which has been described as the archetypal starburst galaxy [17] and as a prototype of superwind galaxies [18]. The galactic magnetic field during particle propagation could certainly account for the required $37^{\circ}$ deflection.

It has been suggested that within this type of galaxy, iron nuclei can be accelerated to extremely high energies if a two step process is invoked [19].

- In a first stage, ions are diffusively accelerated up to a few PeV at single supernova shock waves in the nuclear region of the galaxy [20]. Since the cosmic ray outflow is convection dominated, the typical residence time of the

1) One must, however, always be aware that extensive air shower simulations depend strongly on the hadronic interaction event generator [11]. 
nuclei in the starburst results in $t \sim 1 \times 10^{11} \mathrm{~s}$. Thus, the total path traveled is substantially shorter than the mean free path (which scales as $A^{-2 / 3}$ ) of a super-heavy nucleus (for details see [19]).

- Those which are able to escape from the central region without suffering catastrophic interactions could be eventually re-accelerated to superhigh energies at the terminal shocks of galactic superwinds generated by the starburst. The mechanism efficiently improves as the charge number $Z$ of the particle is increased. For this second step in the acceleration process, the photon field energy density drops to values of the order of the cosmic background radiation (we are now far from the starburst region).

The dominant mechanism for energy losses in the bath of the universal cosmic radiation is the photodisintegration process [21] and has been calculated [22] for ${ }^{197} \mathrm{Au}$, a typical heavy nucleus.

It is straightforward to show that a superheavy nucleus of a few hundred $\mathrm{EeV}$ emitted by M82 can traverse almost unscathed through the primeval radiation to produce an extensive air shower after interaction with the earth atmosphere. Even failing this, the debris of relativistic super-heavy nuclei, which can survive a $100 \mathrm{Mpc}$ journey through the primeval radiation can reach the earth and initiate extensive airshowers, though now the primaries reaching the earth will be of lower mass.

\section{CONCLUSIONS}

The idea that the highest energy cosmic rays might be magnetic monopoles is an exciting one, but a difficult one to test. While extensive airshowers they produce can be quite different from proton or nucleus initiated showers, the details are highly dependent on kinematics - in particular the monopole mass and energy. Very heavy nuclei (or their debris) are also possible, less exotic candidates for the highest energy cosmic rays. Only time and more data will tell whether these speculations are correct or not. More detailed studies can be found in $[10,22,23]$.

\section{ACKNOWLEDGEMENTS}

We would like to thank all our colleagues with the Pierre Auger Observatory. J.S. would like to thank the organizers of the conference for a most stimulating meeting (and lots of tequila!). We would also like to acknowledge the support of the National Science Foundation and CONICET (Argentina). 


\section{REFERENCES}

1. For a recent survey see for instance, S. Yoshida and H. Dai, J. Phys. G 24 (1998) 905 .

2. K. Greisen, Phys. Rev. Lett. 16 (1966) 748; G. T. Zatsepin, and V. A. Kuz'min, Pis'ma Zh. Éksp. Teor. Fiz. 4 (1966) 114 [JETP Lett. 4, 78 (1966)].

3. N. A. Porter, Nuovo. Cim., 16 (1960) 958.

4. T. W. Kephart, and T. J. Weiler, Astropart. Phys 4 (1996) 271.

5. G. 't Hooft, Phys. Rev. Lett. 37 (1976) 8.

6. M. Mattis, and E. Mottola (eds.), "Baryon Number Violation at the SSC?", Proceedings of the Santa Fe Workshop, 27 - 30 April, 1990 (Singapore: World Scientific).

7. B. Andersson et al., Phys. Rep. 97 (1983) 31.

8. S. J. Sciutto, http://www.fisica.unlp.edu.ar/auger/aires/ppal.html

9. C. O. Escobar and R. A. Vazquez, Astropart. Phys. 10 (1999) 197.

10. L. Anchordoqui et al., [astro-ph/0007403].

11. L. A. Anchordoqui, M. T. Dova and S. J. Sciutto, in Proc. 26 $6^{\text {th }}$ International Cosmic Ray Conference, (Eds. D. Kieda, M. Salamon and B. Dingus, Salt Lake City, Utah, 1999) vol. I, p. 147; L. A. Anchordoqui, M. T. Dova, L. N. Epele and S. J. Sciutto, Phys. Rev. D59 (1999) 094003.

12. E. M. Burbidge, G. R. Burbidge, W. A. Fowler, and F. Hoyle, Rev. Mod. Phys. 29 (1957) 547.

13. F. Halzen, R. A. Vázquez, T. Stanev, and H. P. Vankov, Astropart. Phys. 3 (1995) 151.

14. S. Sakai and B. Madore, Detection of the Red Giant Branch Stars in M82 Using the Hubble Space Telescope, [astro-ph/9906484].

15. See for instance, T. W. B. Muxlow et al., Mont. Not. Roc. Astron. Soc. 266 (1994) 455; J. S. Ulvestad and R. J. Antonucci, Astrophys. J. 448 (1997) 621; D. A. Forbes et al., Astrophys. J. 406 (1993) L11; R. de Grijs et al., Supernova Remnants in the Fossil Starburt in M82, [astro-ph/9909044].

16. J. W. Elbert and P. Sommers, Astrophys. J. 441 (1995) 151.

17. A. J. Fitt and P. Alexander, Mont. Not. Roc. Astron. Soc. 261 (1993) 445.

18. T. H. Heckman, L. Armus and G. K. Miley, Astrophys. J. 74 (1990) 833.

19. L. A. Anchordoqui, G. E. Romero, and J. A. Combi, Phys. Rev. D 60 (1999) 10300.

20. P. O. Lagage and C. J. Cesarsky, Astron. Astrophys. 125 (1983) 249.

21. J. L. Puget, F. W. Stecker and J. H. Bredekamp, Astrophys. J. 205 (1976) 638; L. N. Epele and E. Roulet, J. High Energy Phys. 10 (1998) 009; F. W. Stecker, and M. H. Salamon, Astrophys. J. 512 (1999) 521.

22. L. Anchordoqui et al., Phys. Lett. B482 (2000) 343.

23. M. T. Dova and J. Swain, Proceedings of the 26th International Cosmic Ray Conference, Utah, 1999, vol 2, 336 . 